\title{
Emotion Recognition using Parabolic Phase Space Mapping for Heart Rate Variability Analysis
}

\author{
Shahab Rezaei ${ }^{1}$, Sadaf Moharreri ${ }^{2}$, Shadi Ghiasi ${ }^{3}$, Saman Parvaneh ${ }^{4,5}$ \\ ${ }^{1}$ Islamic Azad University, Central Tehran Branch, Tehran, Iran \\ ${ }^{2}$ Islamic Azad University, Khomeini Shahr Branch, Isfahan, Iran \\ ${ }^{3}$ K.N. Toosi University of Technology, Tehran, Iran \\ ${ }^{4}$ Islamic Azad University, Science and Research Branch, Tehran, Iran \\ ${ }^{5}$ Philips Research North America, Cambridge, MA, USA
}

\begin{abstract}
In this paper, extracted features from Parabolic Phase Space Mapping (PPSM) of RR time series and its extended version were used to distinguish four emotionstates (energetic, calm, happy, and anxious) induced by picture stimuli. In order to induce emotions, four groups of pictures with different emotional concepts were used as a visual stimulus while the lead II of ECG was recorded. Induced emotion was confiremed by self-assessment manikin test. The results show that extracted features were significantly different between energetic and calm, energetic and happy, energetic and anxious, calm and happy, calm and anxious, and happy and anxious $(p<0.05)$.
\end{abstract}

\section{Introduction}

A mental state that arises spontaneously rather than through conscious effort is called emotion [1] and is often accompanied by physiological changes. It is shown that one approach for evaluating physiological response to emotions is heart rate variability (HRV) analysis [1,2]. $\mathrm{HRV}$ is responsible for representing the changes on automatic nervous system (ANS), especially in the activation and deactivation of sympathetic and parasympathetic systems.

The people explain their emotions differently, so we have to had scales for measuring and labeling different emotions in different people [3] which are valence and arousal [4]. Valence represents the pleasantness of stimuli, with positive (or pleasant) at one end and negative (or unpleasant) at the other [4]. Another dimension is arousal (activation level). Researches shown that some emotions can be distinguished depending on their arousal and valence level [3, 4]. In this study, the arousal and valence dimension of SAM test were used to estimate emotions which are induced by visual stimuli using $2 \mathrm{D}$ model of emotions.

Furthermore, HRV analysis using the nonlinear maps (Parabolic Phase Space Mapping (PPSM) and its extended version) was used for studying physiological response to different emotions. The PPSM can highlight subtle changes in RR time series and was successfully used for arrhythmia detection $[5,6]$.

\section{Method and Material}

In this paper, pictures were used for emotion induction. Self-assessment manikin (SAM) test and 2D model of emotions were used to capture induce emotion. Then, the relation between induced emotions and its physiological response was analyzed by the HRV analysis using the novel Parabolic Phase Space Mapping (PPSM) and the extended novel features (Figure 1).

\subsection{Participants and Protocol}

In order to induce four different emotions (energetic, calm, happy, and anxious), four groups of pictures with four different emotional concepts from the international affective picture system (IAPS) [7] were used as a visual stimulus, four pictures in each group. ECG (Lead II with sampling frequency: $200 \mathrm{~Hz}$ ) was recorded from 20 healthy males as subjects (Age: $25.04 \pm 0.87$ ) they were watching each group of pictures (each group was shown for five minutes). ECG data was recorded while participants were seated in a chair in front of a computer monitor and were watching stimuli pictures (presented for five minutes each group). A 10 minutes break was given between each picture stimuli to cancel the effects of previous stimulation. In order to label the emotion state which was induced by 
each picture group, SAM test was used before the break to measure the valence and arousal level of each emotion.

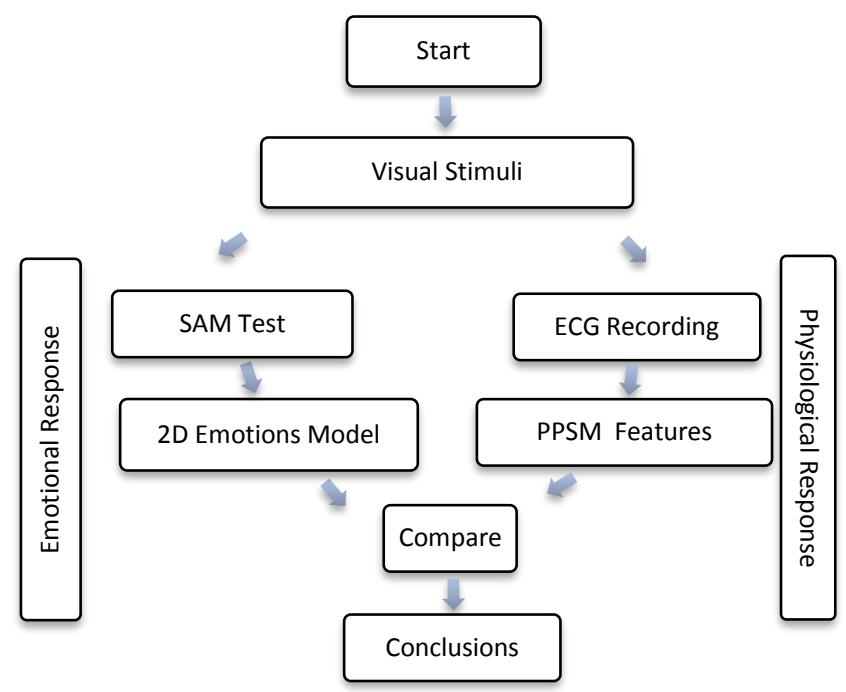

Figure 1. Capturing emotional response to visual stimuli using physiological response.

\subsection{Self-Assessment Manikin Test}

After each stimulus, the subjects answered the SAM test. SAM test consists of a series of pictograms to judge the affective quality of stimuli by arousal and valence level.

As shown in Figure 2, the SAM figures range from frowning, unhappy to smiling, happy, on the valence dimension [8]. For the arousal dimension, the figures range from relaxed, sleepy to excited and wide-eyed [8]. The subject can select any of the five figures comprising each scale $(-2,-1,0,+1,+2)$.

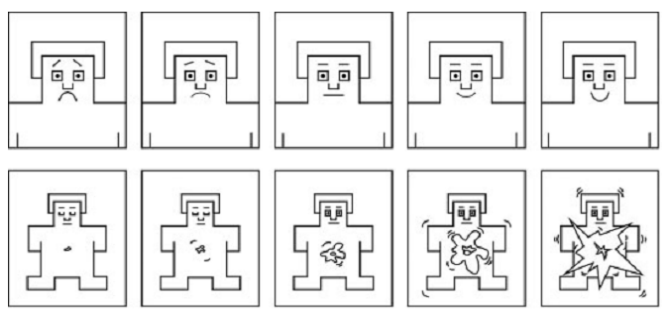

Figure 2. Self- Assessment Manikins (SAM) test

\subsection{D Model of Emotions}

As mentioned in previous section, every emotion is defined by two dimensions Arousal and Valence. By plotting arousal versus valence, a 2D emotion model as it is shown in Figure 3 will be constructed. This model provides a simplified representation of human emotions by two dimensions (arousal and valence) [9].

In this study, we have considered four groups of emotions that are energetic, calm, happy, and anxious.

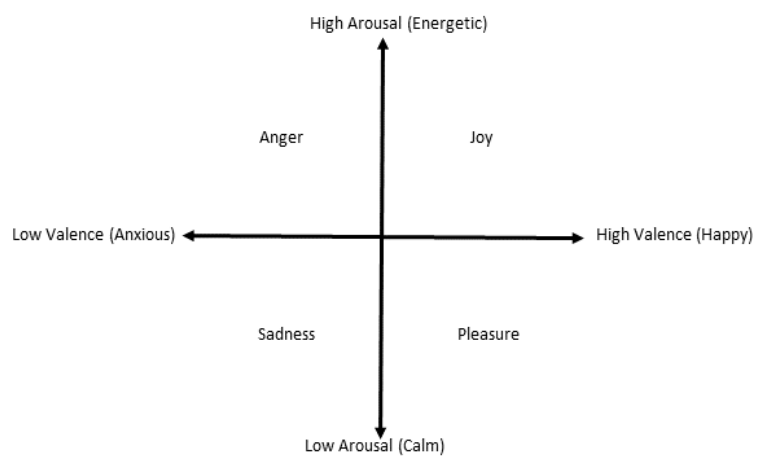

Figure 3. 2D model of emotions

\subsection{HRV Analysis}

For QRS detection, Pan-Tompkins algorithm [10] following by manual inspection was employed. Extracted QRS peaks were used for creating RR intervals.

\subsubsection{Parabolic Phase Space Mapping (PPSM)}

For discriminating heart arrhythmia, Jafarnia Dabanloo et al. introduced a Parabolic Phase space Mapping (PPSM) for analysis of RR intervals [6].

Using $R R$ time series $\left(R_{1}, R_{2}, \ldots, R_{n}, R_{n+1}\right)$, input to PPSM will be created using the following equations:

$$
\begin{aligned}
& \mathrm{x}=\left\{\mathrm{x}_{1}, \mathrm{x}_{2}, \ldots, \mathrm{x}_{\mathrm{n}}\right\}=\left\{\mathrm{RR}_{1}, \mathrm{RR}_{2}, \ldots, \mathrm{RR}_{\mathrm{n}}\right\} \\
& \mathrm{y}=\left\{\mathrm{y}_{1}, \mathrm{y}_{2}, \ldots, \mathrm{y}_{\mathrm{n}}\right\}=\left\{\mathrm{RR}_{2}, \mathrm{RR}_{3}, \ldots, \mathrm{RR}_{\mathrm{n}+1}\right\}
\end{aligned}
$$

Where $n$ is the number of points in the map which is one less than the length of the RR time series $[6,11]$. PPSM consists of all the ordered pairs:

$$
\left(x_{i},\left(\overline{R R}-y_{i}\right)^{2}\right)
$$

in which $i=1,2,3, \ldots, n$ [6]. $R R$ is average of $R R$ intervals defined as:

$$
\operatorname{mean}(R R)=\overline{R R}=\frac{1}{n+1} \sum_{i=1}^{n+1} R R_{i}
$$

By evaluating the distribution of points in PSPM which is shown in Fig. 4, Jafarnia Dabanloo et al. could estimate a two degree polynomial equation in the form of $\mathrm{Y}=$ $A \mathrm{x}^{2}+B \mathrm{x}+C$, in which [6]:

$$
Y=\left(\overline{R R}-y_{i}\right)^{2}
$$


The coefficients of the estimated polynomial $(A, B$, and $C)$ fit the point distribution in PPSM were extracted as features for representing this map [6].

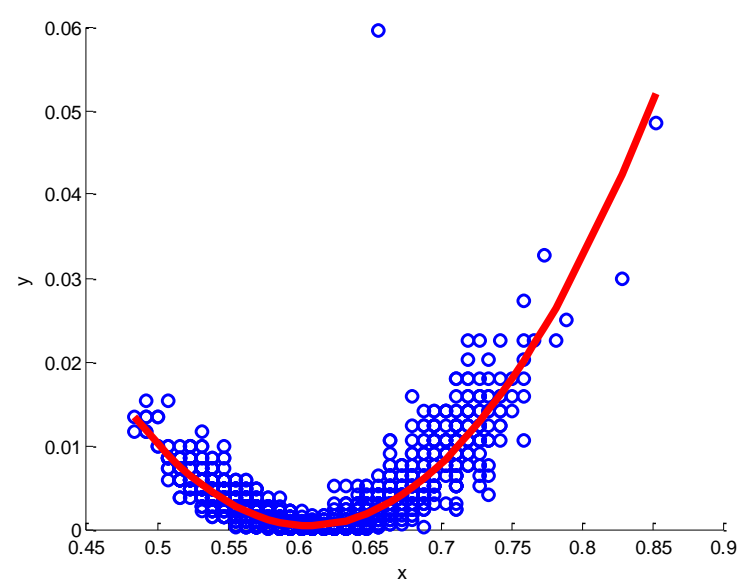

Figure 4. Points' distribution in $P S P M$ and the estimation of a quadratic equation.

\subsubsection{Extended Parabolic Phase Space Mapping (EPPSM)}

In EPPSM, new features are extracted based on the location of point's distribution relative to the estimated polynomial. Using the following equations, the points will be categorized as a) points which are above the curve (A), b) points which are on the curve $(\mathrm{O})$, and c) points which are below the curve (B) (figure 5):

$$
\begin{aligned}
& \text { If }\left(y-A x^{2}-B x-C\right)<0 \Rightarrow p_{i} \in B \\
& \text { If }\left(y-A x^{2}-B x-C\right)=0 \Rightarrow p_{i} \in O \\
& \text { If }\left(y-A x^{2}-B x-C\right)>0 \Rightarrow p_{i} \in A
\end{aligned}
$$

In which $p_{i}\left(x_{i}, y_{i}\right)$ is each point in the mapping and $i=$ $1,2, \ldots, n$.

Compared to PPSM, location of points relative to estimated polynomial will be marked by color in EPPSM (blue, red, and green shows that a point is above, on, and below estimated polynomial, respectively), Figure 5. After finding the classes of all the points, number of points in each class was considered as features in EPPSM $\left(N_{B}, N_{O}\right.$, and $N_{A}$ ). $N_{B}$ is the number of points in class $B$ that means the number of points which are below the curve, $N_{O}$ is the number of points in class $O$ that means the number of points which are on the estimated curve, and $N_{A}$ is the number of points in class $A$ that means the number of points which are above the estimated curve.

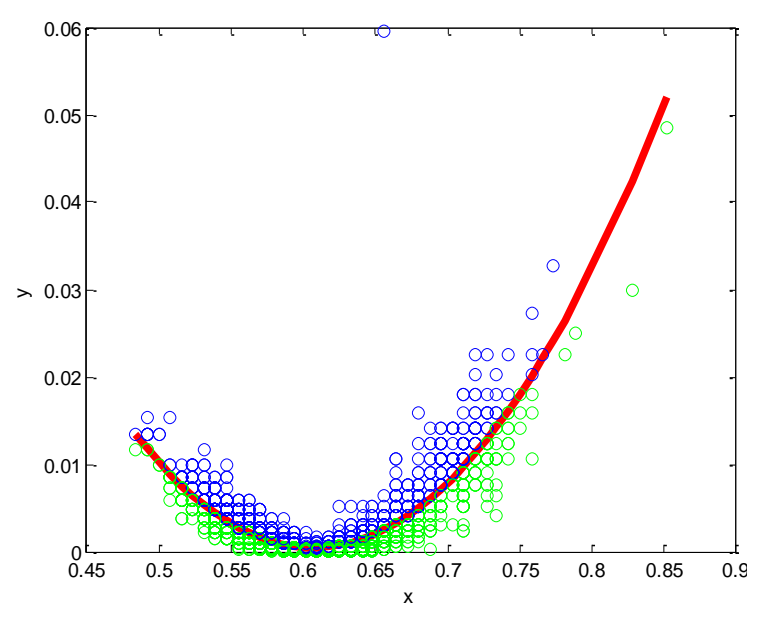

Figure 5. Points in PPSM are color coded based on their position relative to the estimated polynomial.

\subsection{Statistical Analysis}

All continuous features were reported as mean \pm standard deviation. In this study, Kruskal-Wallis test was used to compare proposed features across different emotion groups while significant statistical level was set to 0.05 .

\section{Results}

Means and standard deviations of extracted features across four emotion groups have been reported in Table 1. The $p$-values obtained from Kruskal-Wallis analysis are summarized in Table 2 and Table 3 for all six extracted features across different emotion groups.

There were a significant differences in A between energetic and happy (p-value $<0.05)$ and between happy and anxious ( $\mathrm{p}$-value $<0.05$ ). Furthermore, feature $\mathrm{C}$ was only significantly different between calm and anxious. In features extracted from EPPSM, $N_{B}$ was significantly different between energetic and calm, calm and happy, and calm and anxious (p-value<0.05). Also, $N_{A}$ was significantly different between energetic and calm, energetic and happy, energetic and anxious, calm and happy, calm and anxious, and happy and anxious (pvalue $<0.01)$.

Table 1. Means and standard deviations of extracted features across four groups of emotions

\begin{tabular}{ccccc} 
& \multicolumn{4}{c}{ Emotions } \\
\cline { 2 - 5 } & Energetic & Calm & Happy & Anxious \\
\hline$A$ & $0.57 \pm 0.38$ & $-0.01 \pm 0.11$ & $0.05 \pm 0.17$ & $0.26 \pm 0.14$ \\
\hline$B$ & $-0.80 \pm 0.56$ & $-0.63 \pm 0.99$ & $-2.95 \pm 9.28$ & $-0.47 \pm 0.31$ \\
\hline$C$ & $0.29 \pm 0.21$ & $2.28 \pm 8.26$ & $32.99 \pm 119.72$ & $0.23 \pm 0.19$ \\
\hline$N_{B}$ & $2478 \pm 457$ & $53 \pm 87$ & $2255 \pm 202$ & $2316 \pm 319$ \\
\hline$N_{O}$ & $0 \pm 0$ & $0 \pm 0$ & $0 \pm 0$ & $0 \pm 0$ \\
\hline$N_{A}$ & $1148 \pm 457$ & $2 \pm 4$ & $136 \pm 202$ & $842 \pm 319$ \\
\hline
\end{tabular}


Table 2. $P$-value for PPSM features across different emotion groups (values in bold indicate significant differences, $p$-value<0.05).

\begin{tabular}{cccc}
\hline \multirow{2}{*}{ Groups } & \multicolumn{3}{c}{ PPSM Features } \\
\cline { 2 - 4 } & $A$ & $B$ & $C$ \\
\hline Energetic \& Calm & 0.82 & 0.26 & 0.07 \\
Energetic \& Happy & $\mathbf{0 . 0 5}$ & 0.19 & 0.19 \\
Energetic \& Anxious & 0.05 & 0.07 & 0.19 \\
Calm \& Happy & 0.06 & 0.13 & 0.08 \\
Calm \& Anxious & 0.60 & 0.11 & $<\mathbf{0 . 0 5}$ \\
Happy \& Anxious & $\mathbf{0 . 0 5}$ & 0.96 & 0.16 \\
\hline
\end{tabular}

Table 3. $P$-value for EPPSM Features across different emotion groups (values in bold indicate significant differences, $p$-value $<0.05$ ).

\begin{tabular}{cccc}
\hline Groups & \multicolumn{3}{c}{ EPPSM Features } \\
\cline { 2 - 4 } & $N_{B}$ & $N_{O}$ & $N_{A}$ \\
\hline Energetic \& Calm & $\mathbf{0 . 0 5}$ & - & $<\mathbf{0 . 0 5}$ \\
Energetic \& Happy & 0.39 & - & $<\mathbf{0 . 0 5}$ \\
Energetic \& Anxious & 0.56 & - & $\mathbf{0 . 0 1}$ \\
Calm \& Happy & $<\mathbf{0 . 0 5}$ & - & $<\mathbf{0 . 0 5}$ \\
Calm \& Anxious & $\mathbf{0 . 0 5}$ & - & $<\mathbf{0 . 0 5}$ \\
Happy \& Anxious & 0.78 & - & $<\mathbf{0 . 0 5}$ \\
\hline
\end{tabular}

\section{Discussion}

In this paper, parabolic phase space mapping and its extended version were created using RR intervals. Extracted features from these two mappings were significantly different between four emotional states induced by standardize pictures stimuli. Results suggests that HRV analysis using these mappings and associated parameters can capture changes in physiological response to emotion induced by pictures stimuli.

\section{References}

[1] A. Haag, S. Goronzy, P. Schaich, and J. Williams, "Emotion Recognition using Bio-Sensors: First Steps towards an Automatic System," Affective Dialogue Systems, pp. 36-48, 2004.

[2] S. Parvaneh, G. S. Grewal, E. Grewal, R. A. Menzies, T. K. Talal, D. G. Armstrong, et al., "Stressing the dressing: Assessing stress during wound care in real-time using wearable sensors," Wound Medicine, vol. 4, pp. 21-26, 2014.

[3] S. Moharreri, S. Parvaneh, N. Jafarnia Dabanloo, and A. M. Nasrabadi, "Utilizing Occurrence Sequence of Heart Rate's Phase Space Points in order to Discriminate Heart Arrhythmia," in the 17th Iranian Conference of Biomedical Engineering (ICBME2010), Isfahan, Iran, 2010.

[4] K. Kim, S. Bang, and S. Kim, "Emotion recognition system using short-term monitoring of physiological signals," Medical and biological engineering and computing, vol. 42, pp. 419-427, 2004.

[5] S. Moharreri, S. Rezaei, N. Jafarnia Dabanloo, and S. Parvaneh, "Extended Parabolic Phase Space Mapping (EPPSM): Novel Quadratic Function for Representation of Heart Rate Variability Signal," presented at the Computing in Cardiology (CinC 2014), 2014.

[6] N. Jafarnia Dabanloo, S. Moharreri, S. Parvaneh, and A. M. Nasrabadi, "Application of Novel Mapping for Heart Rate Phase Space and Its Role in Cardiac Arrhythmia Diagnosis," presented at the Computing in Cardiology (CinC 2010), 2010.

[7] P. Lang, M. Bradley, and B. Cuthbert, "International affective picture system (IAPS): digitized photographs, instruction manual and affective ratings," G. University of Florida, Ed., ed, 2005.

[8] H. Suk and H. Irtel, "Emotional response to color across media," Color Research \& Application, vol. 35, pp. 64-77, 2010. [9] J. Kim and E. André, "Emotion recognition based on physiological changes in music listening," IEEE Transactions on Pattern Analysis and Machine Intelligence, pp. 2067-2083, 2008. [10] W. J. T. J. Pan, "A Real Time QRS Detection Algorithm," IEEE Trans Biomed Eng, vol. 32(3), pp. 230-236, 1985.

[11] J. Piskorski and P. Guzik, "Geometry of the Poincaré plot of RR intervals and its asymmetry in healthy adults," Physiological measurement, vol. 28, p. 287, 2007.

Address for correspondence,

Saman Parvaneh

2 Canal Park, 3rd floor, Cambridge, MA 02141

parvaneh@ieee.org 